Article

\title{
Organocatalytic Asymmetric Aldol Reaction of Arylglyoxals and Hydroxyacetone: Enantioselective Synthesis of 2,3-Dihydroxy-1,4-diones
}

\author{
Yu-Hao Zhou, Yu-Zu Zhang, Zhu-Lian Wu, Tian Cai, Wei Wen * (1) and Qi-Xiang Guo * \\ Key Laboratory of Applied Chemistry of Chongqing Municipality, School of Chemistry and Chemical \\ Engineering, Southwest University, Chongqing 400715, China; zyh8182868@email.swu.edu.cn (Y.-H.Z.); \\ zhyzlongzhz@126.com (Y.-Z.Z.); wzl12@swu.edu.cn (Z.-L.W.); caitian@swu.edu.cn (T.C.) \\ * Correspondence: wenwei1989@swu.edu.cn (W.W.); qxguo@swu.edu.cn (Q.-X.G.)
}

Academic Editor: Renata Riva

Received: 29 December 2019; Accepted: 1 February 2020; Published: 3 February 2020

Abstract: A highly efficient quinine-derived primary-amine-catalyzed asymmetric aldol addition of hydroxyacetone to arylglyoxals is described. Structurally diverse anti-2,3-dihydroxy-1,4-diones were generated in high yields, with good diastereoselectivities and enantioselectivities.

Keywords: organocatalysis; aldol reaction; asymmetric synthesis; 2,3-Dihydroxy-1,4-dione; arylglyoxal

\section{Introduction}

The enantioselective aldol reaction is among the most important synthetic tools for $\mathrm{C}-\mathrm{C}$ bond formation in organic synthesis [1-3]. Arylglyoxals are endogenous $\alpha$-oxoaldehydes that have been extensively used as electrophiles in related enantioselective aldol processes to afford synthetically important chiral 2-hydroxy-1,4-dicarbonyl compounds [4-19]. For example, in 2009, Feng and coworkers developed an asymmetric aldol-type reaction between arylglyoxal derivatives and 2-oxindoles catalyzed by an $N, N^{\prime}$-dioxide-Sc(III) complex [4]. Various optically active 3-substituted oxindoles bearing $\alpha$-hydroxyl ketone units were produced in excellent yields, with excellent diastereoselectivities and enantioselectivities. Subsequently, Hayashi and coworkers reported a chiral diarylprolinol-catalyzed direct aldol reaction of glyoxal derivatives with aldehydes, affording chiral 2-hydroxy-1,4-dicarbonyl compounds with good experimental outcomes [6]. Zhao and coworkers also reported an aldol reaction between cyclic ketones and arylglyoxals using a cinchona alkaloid-derived thiourea catalyst [10]. Most recently, Wang and coworkers disclosed an aldol reaction of 2-hydroxyacetophenone with ethyl glyoxylate catalyzed by a dinuclear zinc-azaphenol complex [11]. The desired aldol products were obtained with moderate diastereoselectivities. Several other research groups have also reported corresponding catalytic asymmetric aldol or aldol-type reactions, employing phenylglyoxal hydrate in a single example [12-19]. In general, reported examples have mainly focused on the catalytic asymmetric synthesis of $\alpha$-hydroxyl ketones or 2,3-dihydroxyl esters (Scheme 1a,b), while the catalytic asymmetric construction of 2,3-dihydroxyl carbonyl compounds has yet to be explored. 2,3-Dihydroxyl-1,4-dicarbonyl compounds are known as excellent building blocks for the synthesis of multiple-hydroxyl-containing carbohydrates [20,21]. The direct catalytic asymmetric aldol addition of hydroxyacetone to arylglyoxals is an ideal transformation that can be anticipated to construct such 2,3-dihydroxyl-1,4-carbonyl units (Scheme 1c). Herein, we report a chiral primary amine-catalyzed direct organocatalytic asymmetric aldol reaction of arylglyoxals with hydroxyacetone, which afforded chiral 2,3-dihydroxy-1,4-diones in high yields with good to excellent diastereoselectivities and excellent enantioselectivities. 
Previous works:

a) for $\alpha$-hydroxyl ketone and $\alpha$-hydroxyl aldehyde synthesis

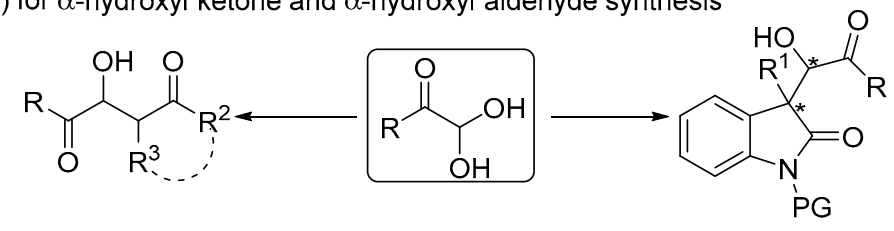

b) for 2,3-dihydroxyl ester synthesis

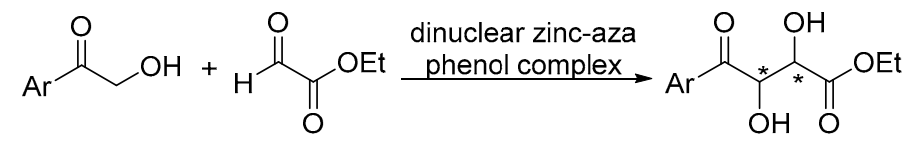

This work:

c) for 2,3-dihydroxyl 1,4-diketone synthesis

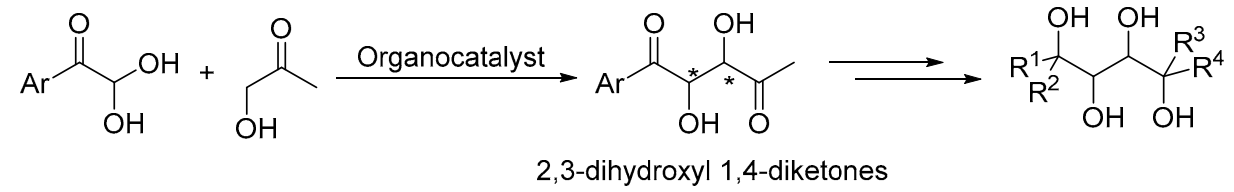

Scheme 1. Catalytic asymmetric aldol reaction of glyoxal derivatives.

\section{Results and Discussion}

\subsection{Optimization of Reaction Conditions}

Phenylglyoxal monohydrate (1a) and hydroxyacetone (2a) were selected as model reactants for initial tests, with various natural amino acids, including L-Proline, L-Serine, and L-Threonine, screened as catalysts [22,23] and N,N-dimethyl formamide (DMF) as solvent. Unfortunately, desired product $4 \mathbf{a}$ was not obtained (Table 1, entries 1-3). Chiral pyrrolidine derivatives $\mathbf{3 a}$ and $\mathbf{3} \mathbf{b}$ were also ineffective in this reaction (entries 4 and 5). Next, chiral trans- $N, N$-dialkyl diaminocyclohexanes $3 \mathbf{c}-3 \mathbf{e}$ were used as catalysts to promote this reaction [24], giving desired product 4a in good yields, with good diastereoselectivities and excellent enantioselectivities (entries 6-8). Catalyst 3f, derived from (1S,2S)-1,2-diphenylethane-1,2-diamine, was also tested, but generated product 4a with poor diastereoselectivity and moderate enantioselectivity (entry 9). The stereoselectivity of 4 a was slightly enhanced when quinine-derived primary amine $3 \mathrm{~g}$ was used as catalyst (84\% yield, 94\% ee, 89:11 dr; entry 10). Based on these results, chiral primary amine $\mathbf{3 g}$ was selected as the best catalyst for further optimization of the reaction conditions. Next, the effect of Brønsted acid additives was studied [25]. When additive 3,5-dinitrobenzoic acid (DNBA) was replaced by a weaker acid, $p$-nitrobenzoic acid, the reaction was slower and the yield, diastereoselectivity, and enantioselectivity of 4 a were decreased slightly (entry 11). Similar results were observed when DNBA was replaced with tosic acid (entry 12). These results indicated that DNBA was the most suitable acid additive for this reaction. Decreasing the reaction temperature clearly enhanced the stereoselectivity. For example, when the reaction was conducted at $0{ }^{\circ} \mathrm{C}$, 4a was obtained with $96 \%$ ee and 93:7 dr (entry 13). With the aim to further enhance the experimental outcome of $4 \mathbf{a}$ by introducing another hydrogen donor group, catalyst $3 \mathbf{h}$ was prepared. However, both the yield and stereoselectivity of 4 a were decreased slightly in the reaction promoted by $3 \mathrm{~h}$ (entry 14). An investigation of the catalyst loading indicated that $10 \mathrm{~mol} \%$ of $\mathbf{3 g}$ was sufficiently effective for this reaction (entry 15). Further decreasing the catalyst loading greatly diminished the reaction outcome (entry 16). Various solvents were examined using catalyst $3 \mathrm{~g}$, with the results indicating that $\mathrm{CHCl}_{3}$ was the best solvent in terms of yield, diastereoselectivity, and enantioselectivity. Based on these results, the reaction conditions depicted in entry 15 were selected as optimal for further substrate scope investigations. 
Table 1. Optimization of reaction conditions ${ }^{a}$.

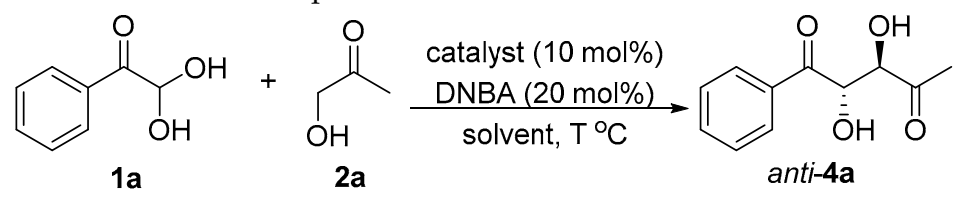

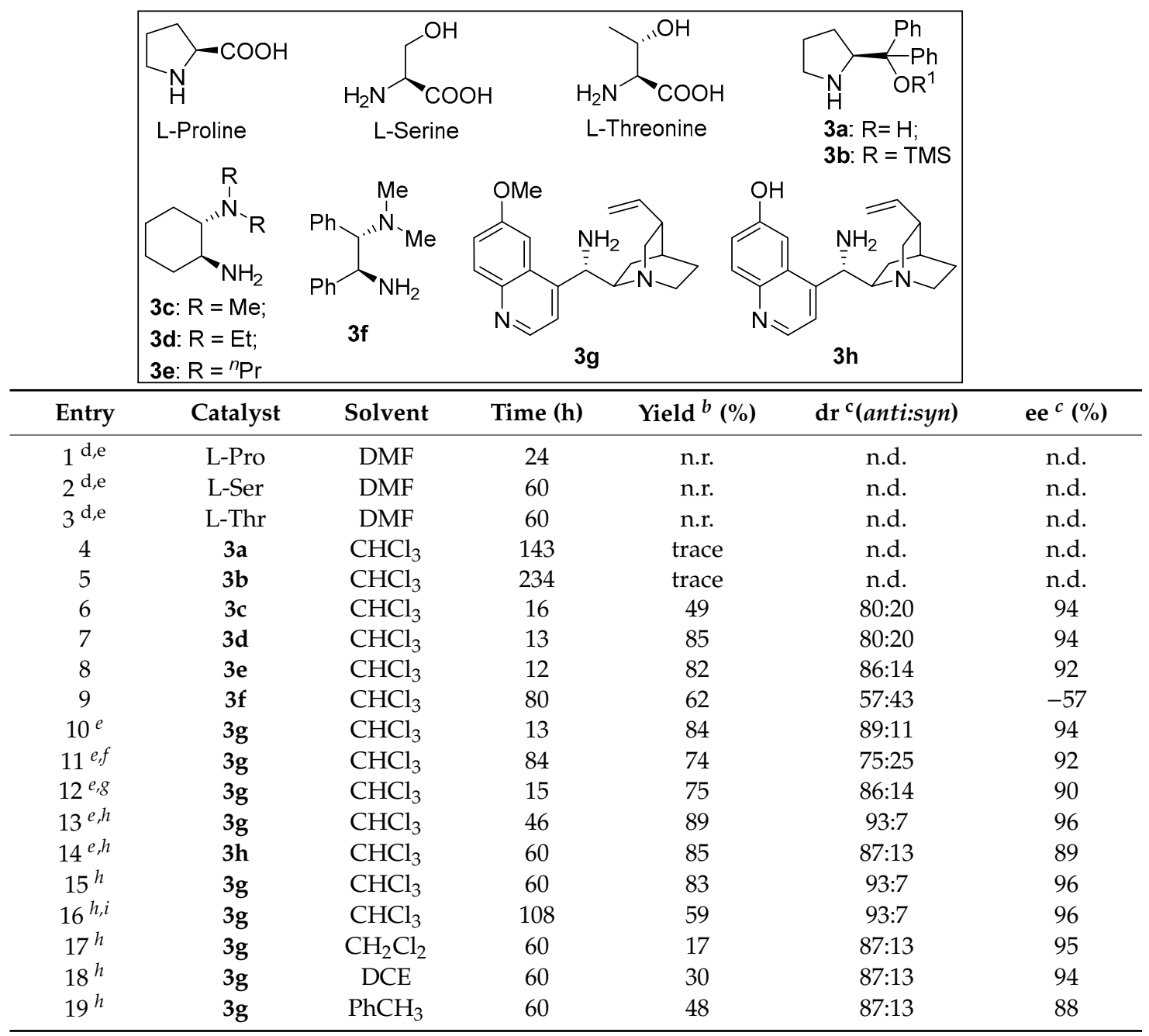

${ }^{a}$ Unless otherwise noted, reactions were performed with $1 \mathrm{a}(0.2 \mathrm{mmol}), 2 \mathrm{a}(1.0 \mathrm{mmol})$, DNBA $(0.02 \mathrm{mmol})$, and catalyst $(0.04 \mathrm{mmol})$ in the specified solvent $(2.0 \mathrm{~mL})$ at $30{ }^{\circ} \mathrm{C} .{ }^{b}$ Isolated yield. ${ }^{c}$ Determined by chiral HPLC. ${ }^{d}$ Reaction performed in the absence of DNBA. ${ }^{e} 20 \mathrm{~mol} \%$ of catalyst used. ${ }^{f} p-\mathrm{NO}_{2} \mathrm{C}_{6} \mathrm{H}_{4} \mathrm{CO}_{2} \mathrm{H}(0.02 \mathrm{mmol})$ used instead of DNBA. ${ }^{g} \mathrm{TsOH}^{\bullet} \mathrm{H}_{2} \mathrm{O}(0.02 \mathrm{mmol})$ used instead of DNBA. ${ }^{h}$ Reaction performed at $0^{\circ} \mathrm{C} .{ }^{i} 5 \mathrm{~mol} \%$ of $3 \mathrm{~g}$ used. $\mathrm{DNBA}=3,5$-dinitrobenzoic acid; $\mathrm{DCE}=1,2$-dichloroethene; n.r. $=$ no reaction; .d. $=$ not determined.

\subsection{Substrate Scope Study}

Under the optimized reaction conditions, anti-selective aldol reactions of hydroxyacetone $2 \mathbf{a}$ with various phenylglyoxals $\mathbf{1 b} \mathbf{b} \mathbf{- 1} \mathbf{p}$ were examined (Figure 1 ). In addition to phenylglyoxal monohydrate $\mathbf{1 a}$, substituted arylglyoxal monohydrates were found to be good reaction partners in this transformation. The electronic properties and positions of substituents on the phenyl ring had almost no influence on the reactivity and stereoselectivity. For example, arylglyoxals bearing 3-Cl-, $\mathrm{Br}-$, or $\mathrm{MeO}-$ substituted phenyl groups reacted with hydroxyacetone smoothly to give the corresponding 2,3-dihydroxy-1,4-diketone products $4 \mathbf{b}-\mathbf{4 m}$ in high yields (82-92\%) with high enantioselectivities (86-93\% ee). For arylglyoxals containing para-substituted phenyl groups, both electron-deficient and electron-rich phenyl-substituted substrates afforded products with excellent yields, diastereoselectivities, and enantioselectivities (Figure 1, 4e-4k). Arylglyoxals bearing disubstituted phenyls were also good reaction partners, 
affording products 41-4o with excellent experimental outcomes. 6-Methoxy-2-naphthyl-substituted arylglyoxal reacted with $\mathbf{2 a}$ to giving product $4 \mathbf{p}$ in excellent yield $(86 \%)$, with high diastereoselectivity (89:11 dr) and excellent enantioselectivity (95\% ee). We also explored other hydroxy ketones, including $\alpha$-hydroxyacetophenone, 1,3-dihydroxyacetone, and ketal-protected 1,3-dihydroxyacetone as donors, which reacted with $\mathbf{1 a}$ to obtain $\mathbf{4 q} \mathbf{- 4}$, but failed. Although all arylglyoxals tested in this reaction gave excellent experimental outcomes, the substrate scope of this reaction could not be expanded further owing to the limited available of arylglyoxal and $\alpha$-hydroxyketone starting materials.

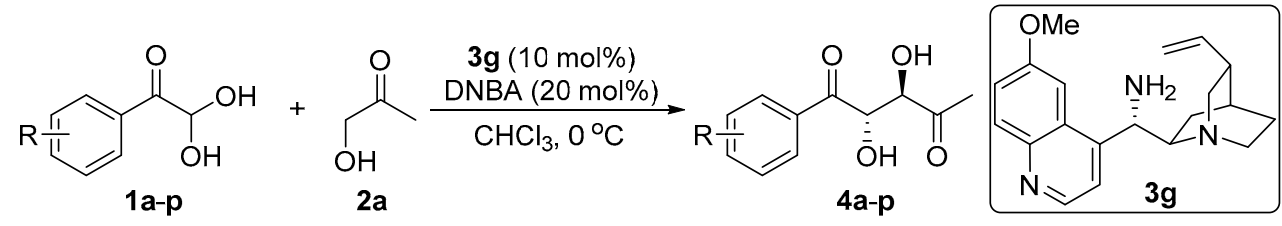<smiles>CC(=O)C(O)C(O)C(=O)c1ccccc1</smiles>

4a, $60 \mathrm{~h}, 83 \%$ yield, $92: 8 \mathrm{dr}, 96 \%$ ee<smiles>CC(=O)C(O)C(O)C(=O)c1ccc(F)cc1</smiles>

4e, $40 \mathrm{~h}, 87 \%$ yield, 90:10 dr, $96 \%$ ee<smiles>CC(=O)C(O)C(O)C(=O)c1cccc(Cl)c1</smiles>

4b, 24h, $82 \%$ yield, $78: 22 \mathrm{dr}, 90 \%$ ee<smiles>CC(=O)C(O)[C@H](O)C(=O)c1cccc(Br)c1</smiles>

4c, $55 \mathrm{~h}, 86 \%$ yield, $81: 19 \mathrm{dr}, 95 \%$ ee<smiles>COc1cccc(C(=O)C(O)C(O)C(C)=O)c1</smiles>

4d, $74 \mathrm{~h}, 90 \%$ yield, $89: 11 \mathrm{dr}, 93 \%$ ee<smiles>CC(=O)C(O)[C@@H](O)C(=O)c1ccc(Cl)c([13CH]=[13CH][13CH3])c1</smiles>

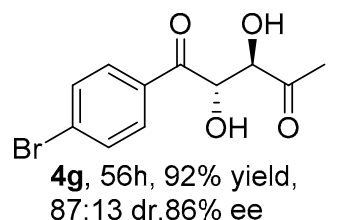<smiles>CC(=O)C(O)[C@@H](O)C(=O)c1ccc(C)cc1</smiles>

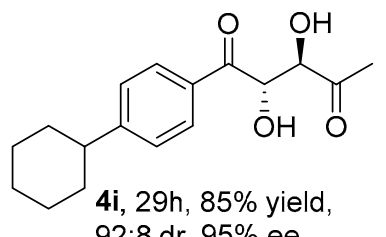<smiles>COc1ccc(C(=O)[C@@H](O)[C@H](O)C(C)=O)cc1</smiles><smiles>CC(=O)C(O)C(O)C(=O)c1ccc(Oc2ccccc2)cc1</smiles><smiles>CC(=O)C(O)C(O)C(=O)c1cc(C(F)(F)F)cc(F)c1F</smiles><smiles>CC(=O)C(O)C(O)C(=O)c1ccc(F)c(F)c1</smiles><smiles>COc1ccc(C(=O)[C@H](O)[C@H](O)C(C)=O)cc1OC</smiles>
$4 \mathrm{n}, 22 \mathrm{~h}, 88 \%$ yield, $92: 8 \mathrm{dr}, 96 \%$ ee<smiles>CC(=O)C(O)[C@@H](O)C(=O)c1cc2c(cc1[18OH])OCO2</smiles>

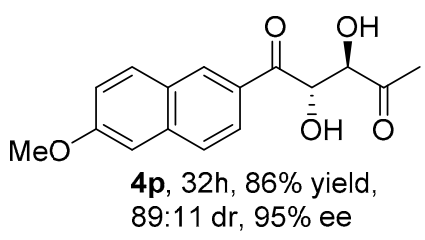<smiles>O=C(c1ccccc1)C(O)C(O)C(=O)c1ccccc1</smiles>

$4 q$, n.r. ${ }^{e}$<smiles>O=C(CO)C(O)[C@H](O)C(=O)c1ccccc1</smiles>

$4 r$, n.r. ${ }^{e}$<smiles>CC1(C)OCC(=O)C([C@H](O)C(=O)c2ccccc2)O1</smiles>

$4 s$, n.r. ${ }^{e}$

Figure 1. Substrate scope study ${ }^{a-d}$. ${ }^{a}$ All reactions were performed with $\mathbf{1}(0.2 \mathrm{mmol}), \mathbf{2 a}(1.0 \mathrm{mmol})$, DNBA $(0.04 \mathrm{mmol})$, and $3 \mathrm{~g}(0.02 \mathrm{mmol})$ in $\mathrm{CHCl}_{3}(2.0 \mathrm{~mL})$ at $0{ }^{\circ} \mathrm{C} .{ }^{b}$ Isolated yield. ${ }^{c} \mathrm{dr}$ determined by ${ }^{1} \mathrm{H}-\mathrm{NMR} .{ }^{d}$ ee of anti-diastereomer determined by chiral HPLC. ${ }^{e}$ n.r. $=$ no reaction. 


\subsection{Scale-Up Experiment and Crystal Structure of Compound $4 j$}

Notably, this reaction was successfully conducted on a 2-mmol scale (Scheme 2), with product $4 \mathbf{j}$ obtained in $86 \%$ yield with $95 \%$ ee and 89:11 dr. The relative and absolute configurations of $4 \mathbf{j}(2 S, 3 R)$ were determined by X-ray crystallography (see the Supplementary Materials) [26]. The stereochemistry of the other aldol products was assigned by comparison with $\mathbf{4 j}$.

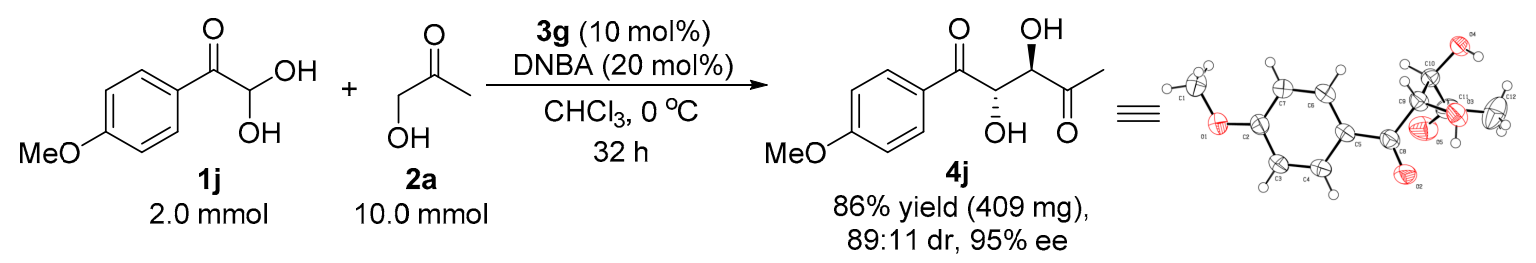

Scheme 2. Scaled-up 2-mmol reaction.

\subsection{Plausible Reaction Mechanism and Transition States}

A proposed catalytic cycle and transition state model [27] are shown in Scheme 3. The condensation of catalyst $\mathbf{3 g}$ with hydroxyacetone $\mathbf{2 a}$ affords imine intermediate $\mathbf{I}$. Intermediate $\mathbf{I}$ then isomerizes to form a (Z)-enamine, with an intramolecular $\mathrm{N}-\mathrm{H} \cdots \mathrm{O}$ hydrogen bond assumed to play a critical role in stabilizing the Z-enamine [23]. Meanwhile, the arylglyoxal is activated by hydrogen-bond formation with the protonated nitrogen atom of the quinuclidine. In the proposed transition state, the Re-face of the enamine attacks the Si-face of the arylglyoxal to give intermediate III. Finally, intermediate III undergoes hydrolysis to afford $4 \mathbf{a}$ with a $2 S, 3 R$-conformation. The Brønsted acid additive facilitates the enamine catalytic cycle.

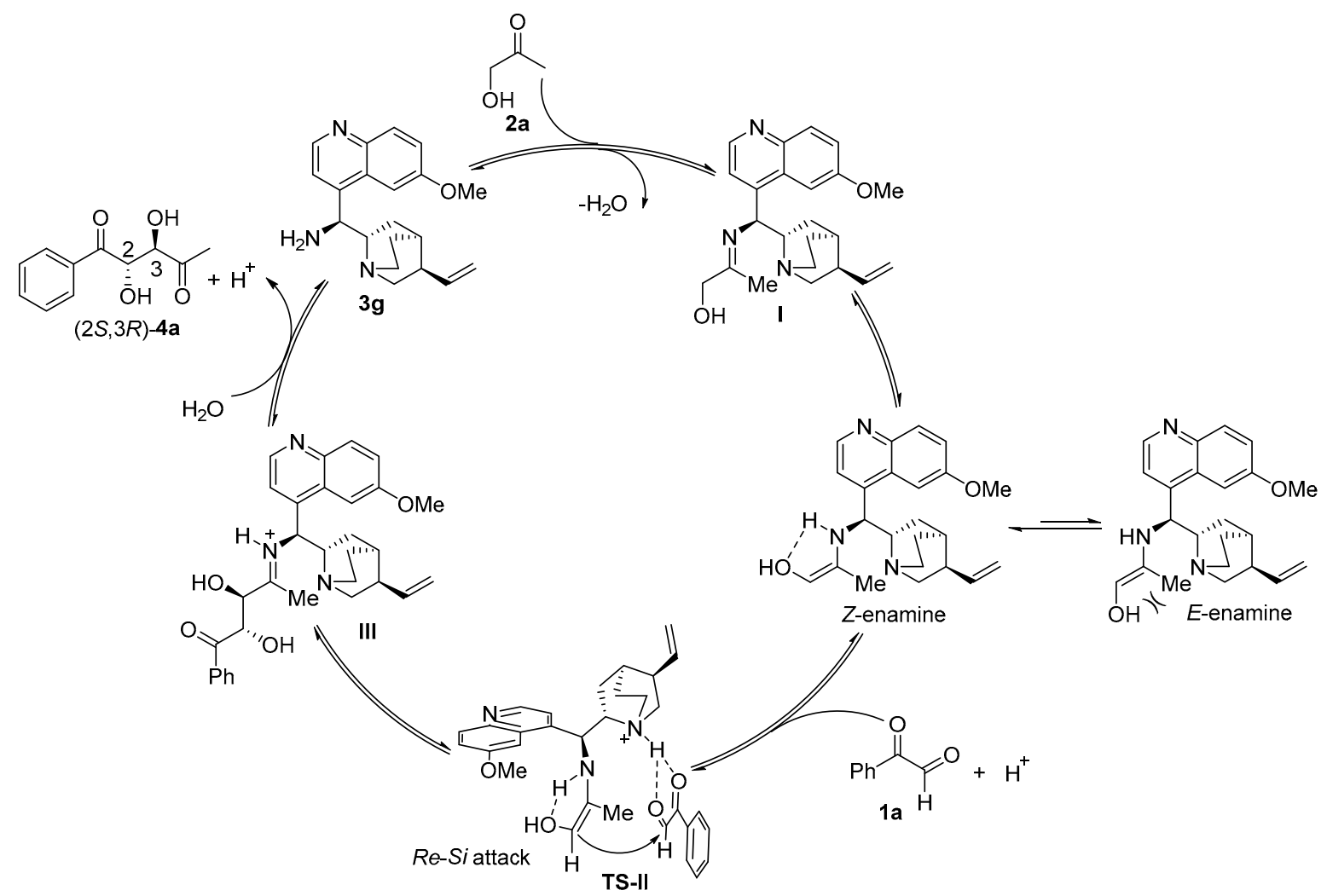

Scheme 3. Plausible reaction mechanism. 


\section{Materials and Methods}

\subsection{General Information}

Unless otherwise noted, commercial reagents were used as received. All reactions were monitored by TLC with silica gel coated plates. ${ }^{1} \mathrm{H}-\mathrm{NMR}(600 \mathrm{MHz})$ and ${ }^{13} \mathrm{C}-\mathrm{NMR}(150 \mathrm{MHz})$ spectra were recorded on Bruker Avance $600 \mathrm{MHz}$ spectrometer. Chemical shifts $(\delta)$ are reported in ppm from tetramethylsilane (TMS) with the solvent resonance as the internal standard. Proton gsignal multiplicities are given as $\mathrm{s}$ (singlet), d (doublet), $\mathrm{t}$ (triplet), q (quartet), m (multiplet), br (broad) or a combination of them. J-values are in Hz. HRMS (ESI-Q-TOF) spectra were recorded on Bruker Impact-II. Enantiomer ratios were determined by HPLC with chiral columns (Chiralpak AD-H, IC-H, IA-H, OD-H, OJ-H columns were purchased from Daicel Chemical Industries, LTD.). Optical rotations were determined at $\lambda=589 \mathrm{~nm}$ (sodium D line) by using a Rudolph-API automatic polarimeter (Hackettstown, NJ, USA).

\subsection{General Procedure for the Synthesis of 2,3-Dihydroxy-1,4-dione Diketone Products $\mathbf{4 a - 4 p}$}

A dry tube was charged with $1(0.2 \mathrm{mmol}), 2(1 \mathrm{mmol})$, catalyst $3 \mathrm{~g}(0.02 \mathrm{mmol})$ and DNBA $(0.04 \mathrm{mmol})$. After addition of $\mathrm{CHCl}_{3}(2.0 \mathrm{~mL})$, the mixture was effectively stirred at $0{ }^{\circ} \mathrm{C}$ and monitored by TLC. After the complete consumption of compound 1, the mixture was concentrated in vacuo and purified by flash chromatography on silica gel $\left(\mathrm{PE}: \mathrm{Et}_{2} \mathrm{O}=1: 1.5\right)$ to afford diastereomeric mixtures of 4a-4p (yield: 82-92\%), see Supplementary Materials.

(2S,3R)-2,3-dihydroxy-1-phenylpentane-1,4-dione. Product 4a was obtained as a white solid in 83\% yield after flash chromatography and the enantiometric excess was determined to be $96 \%$ by HPLC analysis on Daicel Chirapak AD-H column (hexane/isopropanol $=80 / 20$, flow rate $1.0 \mathrm{~mL} / \mathrm{min}, \mathrm{T}=30^{\circ} \mathrm{C}$, $t_{R}$ (major) $9.298 \mathrm{~min}, t_{R}$ (minor) $10.169 \mathrm{~min}$ ), m.p. $53.3-55.3{ }^{\circ} \mathrm{C} ;[\alpha]_{D}^{25}=-31.03\left(\mathrm{c}=0.145, \mathrm{CH}_{2} \mathrm{Cl}_{2}\right)$. The diastereomeric ratio was determined to be 92: 8 by ${ }^{1} \mathrm{H}-\mathrm{NMR}$ on Bruker Avance 600 spectrometer; ${ }^{1} \mathrm{H}-\mathrm{NMR}\left(600 \mathrm{MHz}, \mathrm{CDCl}_{3}\right) \delta 7.97(\mathrm{~d}, J=7.6 \mathrm{~Hz}, 2 \mathrm{H}), 7.64(\mathrm{t}, J=7.4 \mathrm{~Hz}, 1 \mathrm{H}), 7.52(\mathrm{t}, J=7.7 \mathrm{~Hz}$, 2H), $5.28(\mathrm{~s}, 1 \mathrm{H}), 4.47(\mathrm{~s}, 1 \mathrm{H}), 4.05(\mathrm{~d}, J=5.7 \mathrm{~Hz}, 1 \mathrm{H}), 3.94(\mathrm{~d}, J=5.8 \mathrm{~Hz}, 1 \mathrm{H}), 2.13(\mathrm{~s}, 3 \mathrm{H}) ;{ }^{13} \mathrm{C}-\mathrm{NMR}$ $\left(151 \mathrm{MHz}, \mathrm{CDCl}_{3}\right) \delta 207.38,199.04,134.43,129.09,129.00,79.66,75.86,27.43 ;$ HRMS(ESI): calcd. for $\mathrm{C}_{11} \mathrm{H}_{12} \mathrm{NaO}_{4}\left(\mathrm{M}^{+}+\mathrm{Na}\right): 231.0628$, found 231.0628 .

(2S,3R)-1-(3-chlorophenyl)-2,3-dihydroxypentane-1,4-dione. Product $4 \mathbf{b}$ was obtained as a white solid in $82 \%$ yield after flash chromatography and the enantiometric excess was determined to be $90 \%$ by HPLC analysis on Daicel Chirapak IC-H column (hexane/isopropanol = 85/15, flow rate $1.0 \mathrm{~mL} / \mathrm{min}$, $\mathrm{T}=30{ }^{\circ} \mathrm{C}, t_{R}$ (major) $9.905 \mathrm{~min}, t_{R}$ (minor) $10.732 \mathrm{~min}$ ), m.p. $59.1-61.9{ }^{\circ} \mathrm{C} ;[\alpha]_{D}^{25}=-22.78(\mathrm{c}=0.237$, $\mathrm{CH}_{2} \mathrm{Cl}_{2}$ ). The diastereomeric ratio was determined to be 78: 22 by ${ }^{1} \mathrm{H}-\mathrm{NMR}$ on Bruker Avance 600 spectrometer; ${ }^{1} \mathrm{H}-\mathrm{NMR}\left(600 \mathrm{MHz}, \mathrm{CDCl}_{3}\right) \delta 7.95(\mathrm{~s}, 1 \mathrm{H}), 7.84(\mathrm{~d}, J=7.8 \mathrm{~Hz}, 1 \mathrm{H}), 7.64-7.55(\mathrm{~m}, 1 \mathrm{H}), 7.45$ $(\mathrm{t}, J=7.9 \mathrm{~Hz}, 1 \mathrm{H}), 5.17(\mathrm{~d}, J=4.1 \mathrm{~Hz}, 1 \mathrm{H}), 4.41(\mathrm{~d}, J=4.1 \mathrm{~Hz}, 1 \mathrm{H}), 3.91(\mathrm{~d}, J=58.8 \mathrm{~Hz}, 2 \mathrm{H}), 2.21(\mathrm{~s}, 3 \mathrm{H})$; ${ }^{13} \mathrm{C}-\mathrm{NMR}\left(151 \mathrm{MHz}, \mathrm{CDCl}_{3}\right) \delta$ 207.51, 197.96, 136.07, 135.29, 134.07, 130.16, 128.90, 127.01, 79.19, 75.53, 27.31. HRMS(ESI): calcd. for $\mathrm{C}_{11} \mathrm{H}_{11} \mathrm{ClNaO}_{4}\left(\mathrm{M}^{+}+\mathrm{Na}\right): 265.0238$, found 265.0234 .

(2S,3R)-1-(3-bromophenyl)-2,3-dihydroxypentane-1,4-dione. Product 4c was obtained as a white solid in $86 \%$ yield after flash chromatography and the enantiometric excess was determined to be $95 \%$ by HPLC analysis on Daicel Chirapak AD-H column (hexane/isopropanol = 70/30, flow rate $1.0 \mathrm{~mL} / \mathrm{min}$, $\mathrm{T}=30{ }^{\circ} \mathrm{C}, t_{R}$ (major) $6.140 \mathrm{~min}, t_{R}$ (minor) $4.920 \mathrm{~min}$ ), m.p. $66.5-68.1{ }^{\circ} \mathrm{C} ;[\alpha]_{D}^{25}=-14.91(\mathrm{c}=0.248$, $\mathrm{CH}_{2} \mathrm{Cl}_{2}$ ). The diastereomeric ratio was determined to be 81: 19 by ${ }^{1} \mathrm{H}-\mathrm{NMR}$ on Bruker Avance 600 spectrometer; ${ }^{1} \mathrm{H}-\mathrm{NMR}\left(600 \mathrm{MHz}, \mathrm{CDCl}_{3}\right) \delta 8.11(\mathrm{~s}, 1 \mathrm{H}), 7.89(\mathrm{~d}, J=7.7 \mathrm{~Hz}, 1 \mathrm{H}), 7.76(\mathrm{~d}, J=7.8 \mathrm{~Hz}$, $1 \mathrm{H}), 7.40(\mathrm{t}, J=7.9 \mathrm{~Hz}, 1 \mathrm{H}), 5.16(\mathrm{~s}, 1 \mathrm{H}), 4.41(\mathrm{~s}, 1 \mathrm{H}), 3.86(\mathrm{~d}, J=61.5 \mathrm{~Hz}, 2 \mathrm{H}), 2.21(\mathrm{~s}, 3 \mathrm{H}) ;{ }^{13} \mathrm{C}-\mathrm{NMR}$ $\left(151 \mathrm{MHz}, \mathrm{CDCl}_{3}\right) \delta$ 207.43, 197.88, 137.05, 136.22, 131.83, 130.40, 127.47, 123.22, 79.18, 75.42, 27.36. HRMS(ESI): calcd. For $\mathrm{C}_{11} \mathrm{H}_{11} \mathrm{BrNaO}_{4}\left(\mathrm{M}^{+}+\mathrm{Na}\right)$ : 308.9733, found 308.9735. 
(2S,3R)-2,3-dihydroxy-1-(3-methoxyphenyl)pentane-1,4-dione. Product 4d was obtained as an oil in 90\% yield after flash chromatography and the enantiometric excess was determined to be $93 \%$ by HPLC analysis on Daicel Chirapak AD-H column (hexane/isopropanol $=70 / 30$, flow rate $1.0 \mathrm{~mL} / \mathrm{min}, \mathrm{T}=30^{\circ} \mathrm{C}$, $t_{R}$ (major) $7.784 \mathrm{~min}, t_{R}$ (minor) $9.664 \mathrm{~min}$ ), $[\alpha]_{D}^{25}=-4.73\left(\mathrm{c}=0.169, \mathrm{CH}_{2} \mathrm{Cl}_{2}\right.$ ). The diastereomeric ratio was determined to be 89: 11 by ${ }^{1} \mathrm{H}-\mathrm{NMR}$ on Bruker Avance 600 spectrometer; ${ }^{1} \mathrm{H}-\mathrm{NMR}(600 \mathrm{MHz}$, $\left.\mathrm{CDCl}_{3}\right) \delta 7.54(\mathrm{~d}, J=7.6 \mathrm{~Hz}, 1 \mathrm{H}), 7.49(\mathrm{~s}, 1 \mathrm{H}), 7.42(\mathrm{t}, J=7.9 \mathrm{~Hz}, 1 \mathrm{H}), 7.18(\mathrm{dd}, J=8.2,2.3 \mathrm{~Hz}, 1 \mathrm{H}), 5.25$ $(\mathrm{d}, J=2.6 \mathrm{~Hz}, 1 \mathrm{H}), 4.49(\mathrm{~d}, J=3.0 \mathrm{~Hz}, 1 \mathrm{H}), 3.87(\mathrm{~s}, 3 \mathrm{H}), 2.13(\mathrm{~s}, 3 \mathrm{H}) ;{ }^{13} \mathrm{C}-\mathrm{NMR}\left(151 \mathrm{MHz}, \mathrm{CDCl}_{3}\right) \delta$ 207.26, 198.68, 160.05, 135.52, 129.96, 121.32, 120.77, 113.18, 79.64, 75.86, 55.53, 27.31. HRMS(ESI): calcd. for $\mathrm{C}_{12} \mathrm{H}_{14} \mathrm{NaO}_{5}\left(\mathrm{M}^{+}+\mathrm{Na}\right): 261.0733$, found 261.0730 .

(2S,3R)-1-(4-fluorophenyl)-2,3-dihydroxypentane-1,4-dione. Product 4e was obtained as a white solid in $87 \%$ yield after flash chromatography and the enantiometric excess was determined to be $96 \%$ by HPLC analysis on Daicel Chirapak AD-H column (hexane/isopropanol = 80/20, flow rate $1.0 \mathrm{~mL} / \mathrm{min}$, $\mathrm{T}=30{ }^{\circ} \mathrm{C}, t_{R}$ (major) $8.384 \mathrm{~min}, t_{R}$ (minor) $8.987 \mathrm{~min}$ ), m.p. $67.2-69.3^{\circ} \mathrm{C} ;[\alpha]_{D}^{25}=-31.62(\mathrm{c}=0.117$, $\mathrm{CH}_{2} \mathrm{Cl}_{2}$ ) The diastereomeric ratio was determined to be 90: 10 by ${ }^{1} \mathrm{H}-\mathrm{NMR}$ on Bruker Avance 600 spectrometer; ${ }^{1} \mathrm{H}-\mathrm{NMR}\left(600 \mathrm{MHz}, \mathrm{CDCl}_{3}\right) \delta 8.03(\mathrm{dd}, J=7.9,5.7 \mathrm{~Hz}, 2 \mathrm{H}), 7.19(\mathrm{t}, J=8.4 \mathrm{~Hz}, 2 \mathrm{H}), 5.19$ $(\mathrm{s}, 1 \mathrm{H}), 4.41(\mathrm{~s}, 1 \mathrm{H}), 3.99(\mathrm{~d}, J=6.6 \mathrm{~Hz}, 1 \mathrm{H}), 3.88(\mathrm{~d}, J=6.6 \mathrm{~Hz}, 1 \mathrm{H}), 2.19(\mathrm{~s}, 3 \mathrm{H}) ;{ }^{13} \mathrm{C}-\mathrm{NMR}(151 \mathrm{MHz}$, $\left.\mathrm{CDCl}_{3}\right) \delta$ 207.61, 197.59, 167.39, 165.68, 131.98, 131.92, 116.40, 116.25, 79.43, 75.49, 27.50. HRMS(ESI): calcd. for $\mathrm{C}_{11} \mathrm{H}_{11} \mathrm{NaO}_{4}\left(\mathrm{M}^{+}+\mathrm{Na}\right)$ : 249.0534, found: 249.0534 .

(2S,3R)-1-(4-chlorophenyl)-2,3-dihydroxypentane-1,4-dione. Product $4 \mathbf{f}$ was obtained as a white solid in $83 \%$ yield after flash chromatography and the enantiometric excess was determined to be $90 \%$ by HPLC analysis on Daicel Chirapak IA-H column (hexane/isopropanol $=80 / 20$, flow rate $1.0 \mathrm{~mL} / \mathrm{min}$, $\mathrm{T}=30{ }^{\circ} \mathrm{C}, t_{R}$ (major) $9.256 \mathrm{~min}, t_{R}$ (minor) $9.740 \mathrm{~min}$ ), m.p. 81.0-83.1 ${ }^{\circ} \mathrm{C} ;[\alpha]_{D}^{25}=-10.97(\mathrm{c}=0.164$, $\mathrm{CH}_{2} \mathrm{Cl}_{2}$ ). The diastereomeric ratio was determined to be 91: 9 by ${ }^{1} \mathrm{H}-\mathrm{NMR}$ on Bruker Avance 600 spectrometer; ${ }^{1} \mathrm{H}-\mathrm{NMR}\left(600 \mathrm{MHz}, \mathrm{CDCl}_{3}\right) \delta 7.93(\mathrm{dd}, J=5.4,3.1 \mathrm{~Hz}, 2 \mathrm{H}), 7.49(\mathrm{dd}, J=5.4,3.1 \mathrm{~Hz}, 2 \mathrm{H})$, $5.16(\mathrm{~s}, 1 \mathrm{H}), 4.38(\mathrm{~s}, 1 \mathrm{H}), 3.98-3.71(\mathrm{~m}, 2 \mathrm{H}), 2.20(\mathrm{~s}, 3 \mathrm{H}) ;{ }^{13} \mathrm{C}-\mathrm{NMR}\left(151 \mathrm{MHz}, \mathrm{CDCl}_{3}\right) \delta$ 207.40, 197.92, $140.94,132.73,130.37,129.28,79.22,75.31,27.37$. HRMS(ESI): calcd. For $\mathrm{C}_{11} \mathrm{H}_{11} \mathrm{ClNaO}_{4}\left(\mathrm{M}^{+}+\mathrm{Na}\right)$ : 265.0238 , found 265.0232 .

(2S,3R)-1-(4-bromophenyl)-2,3-dihydroxypentane-1,4-dione. Product $4 \mathbf{g}$ was obtained as a white solid in $92 \%$ yield after flash chromatography and the enantiometric excess was determined to be $86 \%$ by HPLC analysis on Daicel Chirapak IA-H column (hexane/isopropanol $=90 / 10$, flow rate $1.0 \mathrm{~mL} / \mathrm{min}$, $\mathrm{T}=30{ }^{\circ} \mathrm{C}, t_{R}$ (major) $18.602 \mathrm{~min}, t_{R}$ (minor) $20.004 \mathrm{~min}$ ), m.p. 98.2-100.2 ${ }^{\circ} \mathrm{C} ;[\alpha]_{D}^{25}=-26.82(\mathrm{c}=0.205$, $\mathrm{CH}_{2} \mathrm{Cl}_{2}$ ). The diastereomeric ratio was determined to be 87: 13 by ${ }^{1} \mathrm{H}-\mathrm{NMR}$ on Bruker Avance 600 spectrometer; ${ }^{1} \mathrm{H}-\mathrm{NMR}\left(600 \mathrm{MHz}, \mathrm{CDCl}_{3}\right) \delta 7.84(\mathrm{dd}, J=8.4,2.1 \mathrm{~Hz}, 2 \mathrm{H}), 7.65(\mathrm{dd}, J=8.1,2.8 \mathrm{~Hz}$, 2H), $5.18(\mathrm{~s}, 1 \mathrm{H}), 4.40(\mathrm{~s}, 1 \mathrm{H}), 4.03(\mathrm{~d}, J=26.9 \mathrm{~Hz}, 1 \mathrm{H}), 3.93(\mathrm{~d}, J=33.1 \mathrm{~Hz}, 1 \mathrm{H}), 2.18(\mathrm{~s}, 3 \mathrm{H}) ;{ }^{13} \mathrm{C}-\mathrm{NMR}$ $\left(151 \mathrm{MHz}, \mathrm{CDCl}_{3}\right) \delta$ 207.66, 198.27, 133.29, 132.39, 130.53, 129.78, 79.37, 75.53, 27.48. HRMS(ESI): calcd for $\mathrm{C}_{11} \mathrm{H}_{11} \mathrm{BrNaO}_{4}\left(\mathrm{M}^{+}+\mathrm{Na}\right)$ : 308.9733, found 308.9732 .

(2S,3R)-2,3-dihydroxy-1-p-tolylpentane-1,4-dione. Product 4h was obtained as a white solid in 89\% yield after flash chromatography and the enantiometric excess was determined to be $97 \%$ by HPLC analysis on Daicel Chirapak AD-H column (hexane/isopropanol $=70 / 30$, flow rate $1.0 \mathrm{~mL} / \mathrm{min}, \mathrm{T}=30^{\circ} \mathrm{C}$, $t_{R}$ (major) $7.000 \mathrm{~min}, t_{R}$ (minor) $7.893 \mathrm{~min}$ ), m.p. $86.1-89.7^{\circ} \mathrm{C} ;[\alpha]_{D}^{25}=-2.49\left(\mathrm{c}=0.281, \mathrm{CH}_{2} \mathrm{Cl}_{2}\right)$. The diastereomeric ratio was determined to be 93: 7 by ${ }^{1} \mathrm{H}-\mathrm{NMR}$ on Bruker Avance 600 spectrometer; ${ }^{1} \mathrm{H}-\mathrm{NMR}\left(600 \mathrm{MHz}, \mathrm{CDCl}_{3}\right) \delta 7.89(\mathrm{~d}, J=8.1 \mathrm{~Hz}, 2 \mathrm{H}), 7.33(\mathrm{~d}, J=7.9 \mathrm{~Hz}, 2 \mathrm{H}), 5.24(\mathrm{~d}, J=3.5 \mathrm{~Hz}, 1 \mathrm{H})$, $4.46(\mathrm{~d}, J=3.5 \mathrm{~Hz}, 1 \mathrm{H}), 3.89(\mathrm{ddd}, J=95.5,59.2,31.9 \mathrm{~Hz}, 2 \mathrm{H}), 2.44(\mathrm{~s}, 3 \mathrm{H}), 2.13(\mathrm{~d}, J=11.8 \mathrm{~Hz}, 3 \mathrm{H})$; ${ }^{13} \mathrm{C}-\mathrm{NMR}\left(151 \mathrm{MHz}, \mathrm{CDCl}_{3}\right) \delta$ 207.03, 198.36, 145.63, 131.62, 129.69, 129.02, 79.64, 75.60, 27.35, 21.78. HRMS(ESI): calcd. for $\mathrm{C}_{12} \mathrm{H}_{14} \mathrm{NaO}_{4}\left(\mathrm{M}^{+}+\mathrm{Na}\right): 245.0784$, found 245.0787 . 
(2S,3R)-1-(4-cyclohexylphenyl)-2,3-dihydroxypentane-1,4-dione. Product 4i was obtained as a white solid in $85 \%$ yield after flash chromatography and the enantiometric excess was determined to be $95 \%$ by HPLC analysis on Daicel Chirapak AD-H column (hexane/isopropanol = 70/30, flow rate $1.0 \mathrm{~mL} / \mathrm{min}$, $\mathrm{T}=30{ }^{\circ} \mathrm{C}, t_{R}$ (major) $7.562 \mathrm{~min}, t_{R}$ (minor) $9.270 \mathrm{~min}$ ), m.p. $66.1-68.1{ }^{\circ} \mathrm{C} ;[\alpha]_{D}^{25}=-11.46(\mathrm{c}=0.218$, $\left.\mathrm{CH}_{2} \mathrm{Cl}_{2}\right)$. The diastereomeric ratio was determined to be 92: 8 by ${ }^{1} \mathrm{H}-\mathrm{NMR}$ on Bruker Avance 600 spectrometer; ${ }^{1} \mathrm{H}-\mathrm{NMR}\left(600 \mathrm{MHz}, \mathrm{CDCl}_{3}\right) \delta 7.92(\mathrm{~d}, J=8.0 \mathrm{~Hz}, 2 \mathrm{H}), 7.35(\mathrm{~d}, J=8.1 \mathrm{~Hz}, 2 \mathrm{H}), 5.24(\mathrm{~d}$, $J=2.8 \mathrm{~Hz}, 1 \mathrm{H}), 4.47(\mathrm{~s}, 1 \mathrm{H}), 3.85(\mathrm{~d}, J=72.9 \mathrm{~Hz}, 2 \mathrm{H}), 2.60(\mathrm{~d}, J=8.4 \mathrm{~Hz}, 1 \mathrm{H}), 2.12(\mathrm{~s}, 3 \mathrm{H}), 1.88(\mathrm{~s}$, $4 \mathrm{H}), 1.78(\mathrm{~d}, J=12.8 \mathrm{~Hz}, 1 \mathrm{H}), 1.43(\mathrm{~d}, J=9.1 \mathrm{~Hz}, 5 \mathrm{H}) ;{ }^{13} \mathrm{C}-\mathrm{NMR}\left(151 \mathrm{MHz}, \mathrm{CDCl}_{3}\right) \delta 206.89,198.39$, $155.49,131.83,129.17,127.57,79.67,75.68,44.86$. 34.03, 27.39, 26.69, 26.01. HRMS(ESI): calcd. for $\mathrm{C}_{11} \mathrm{H}_{22} \mathrm{NaO}_{4}\left(\mathrm{M}^{+}+\mathrm{Na}\right): 313.1410$, found 313.1413 .

(2S,3R)-2,3-dihydroxy-1-(4-methoxyphenyl)pentane-1,4-dione. Product 4j was obtained as an oil in 85\% yield after flash chromatography and the enantiometric excess was determined to be $95 \%$ by HPLC analysis on Daicel Chirapak AD-H column (hexane/isopropanol $=70 / 30$, flow rate $1.0 \mathrm{~mL} / \mathrm{min}, \mathrm{T}=30^{\circ} \mathrm{C}$, $t_{R}$ (major) $8.752 \mathrm{~min}, t_{R}$ (minor) $9.923 \mathrm{~min}$ ). $[\alpha]_{D}^{25}=-16.04\left(\mathrm{c}=0.187, \mathrm{CH}_{2} \mathrm{Cl}_{2}\right.$ ). The diastereomeric ratio was determined to be 92: 8 by ${ }^{1} \mathrm{H}-\mathrm{NMR}$ on Bruker Avance 600 spectrometer; ${ }^{1} \mathrm{H}-\mathrm{NMR}(600 \mathrm{MHz}$, $\left.\mathrm{CDCl}_{3}\right) \delta 7.99(\mathrm{~d}, J=8.8 \mathrm{~Hz}, 2 \mathrm{H}), 6.99(\mathrm{~d}, J=8.8 \mathrm{~Hz}, 2 \mathrm{H}), 5.22(\mathrm{dd}, J=5.7,4.2 \mathrm{~Hz}, 1 \mathrm{H}), 4.45(\mathrm{dd}, J=6.1$, $4.1 \mathrm{~Hz}, 1 \mathrm{H}), 4.05(\mathrm{~d}, J=6.0 \mathrm{~Hz}, 1 \mathrm{H}), 3.95(\mathrm{~d}, J=6.8 \mathrm{~Hz}, 1 \mathrm{H}), 3.89(\mathrm{~s}, 3 \mathrm{H}), 2.13(\mathrm{~s}, 3 \mathrm{H}) ;{ }^{13} \mathrm{C}-\mathrm{NMR}(151$ $\left.\mathrm{MHz}_{,} \mathrm{CDCl}_{3}\right) \delta$ 207.37, 197.17, 164.74, 131.54, 127.06, 114.38, 79.82, 75.48, 55.72, 27.52. HRMS(ESI): calcd for $\mathrm{C}_{12} \mathrm{H}_{14} \mathrm{NaO}_{5}\left(\mathrm{M}^{+}+\mathrm{Na}\right)$ : 261.0736, found 261.0736.

(2S,3R)-2,3-dihydroxy-1-(4-phenoxyphenyl)pentane-1,4-dione. Product 4k was obtained as an oil in 83\% yield after flash chromatography and the enantiometric excess was determined to be $94 \%$ by HPLC analysis on Daicel Chirapak IC-H column (hexane/isopropanol =70/30, flow rate $1.0 \mathrm{~mL} / \mathrm{min}, \mathrm{T}=30^{\circ} \mathrm{C}$, $t_{R}$ (major) $7.932 \mathrm{~min}, t_{R}$ (minor) $8.424 \mathrm{~min}$ ), $[\alpha]_{D}^{25}=-2.08\left(\mathrm{c}=0.048, \mathrm{CH}_{2} \mathrm{Cl}_{2}\right)$. The diastereomeric ratio was determined to be 92: 8 by ${ }^{1} \mathrm{H}-\mathrm{NMR}$ on Bruker Avance 600 spectrometer; ${ }^{1} \mathrm{H}-\mathrm{NMR}(600 \mathrm{MHz}$, $\left.\mathrm{CDCl}_{3}\right) \delta 7.97(\mathrm{~d}, J=8.8 \mathrm{~Hz}, 2 \mathrm{H}), 7.41(\mathrm{t}, J=7.9 \mathrm{~Hz}, 2 \mathrm{H}), 7.22(\mathrm{~s}, 1 \mathrm{H}), 7.08(\mathrm{~d}, J=7.7 \mathrm{~Hz}, 2 \mathrm{H}), 7.02(\mathrm{~d}$, $J=8.8 \mathrm{~Hz}, 2 \mathrm{H}), 5.19(\mathrm{~d}, J=3.4 \mathrm{~Hz}, 1 \mathrm{H}), 4.44(\mathrm{~d}, J=3.7 \mathrm{~Hz}, 1 \mathrm{H}), 2.16(\mathrm{~s}, 3 \mathrm{H}) ;{ }^{13} \mathrm{C}-\mathrm{NMR}(151 \mathrm{MHz}$, $\left.\mathrm{CDCl}_{3}\right) \delta 207.38,197.23,163.22,154.95,131.42,130.17,128.51,125.07,120.47,117.34,79.55,75.38,27.41$. HRMS(ESI): calcd. for $\mathrm{C}_{11} \mathrm{H}_{16} \mathrm{NaO}_{5}\left(\mathrm{M}^{+}+\mathrm{Na}\right)$ : 323.0890, found 323.0886 .

(2S,3R)-1-(2,4-difluorophenyl)-2,3-dihydroxypentane-1,4-dione. Product 41 was obtained as an oil in 87\% yield after flash chromatography and the enantiometric excess was determined to be $97 \%$ by HPLC analysis on Daicel Chirapak AD-H column (hexane/isopropanol $=80 / 20$, flow rate $1.0 \mathrm{~mL} / \mathrm{min}, \mathrm{T}=30^{\circ} \mathrm{C}$, $t_{R}$ (major) $8.257 \mathrm{~min}, t_{R}$ (minor) $9.181 \mathrm{~min}$ ), $[\alpha]_{D}^{25}=-16.08\left(\mathrm{c}=0.143, \mathrm{CH}_{2} \mathrm{Cl}_{2}\right)$ The diastereomeric ratio was determined to be 87: 13 by ${ }^{1} \mathrm{H}-\mathrm{NMR}$ on Bruker Avance 600 spectrometer; ${ }^{1} \mathrm{H}-\mathrm{NMR}(600 \mathrm{MHz}$, $\left.\mathrm{CDCl}_{3}\right) \delta 7.98(\mathrm{dd}, J=15.0,8.3 \mathrm{~Hz}, 1 \mathrm{H}), 7.10-7.00(\mathrm{~m}, 1 \mathrm{H}), 6.95-6.87(\mathrm{~m}, 1 \mathrm{H}), 5.10(\mathrm{~s}, 1 \mathrm{H}), 4.35(\mathrm{~s}, 1 \mathrm{H})$, $4.01(\mathrm{~d}, J=5.5 \mathrm{~Hz}, 1 \mathrm{H}), 3.67(\mathrm{~d}, J=5.8 \mathrm{~Hz}, 1 \mathrm{H}), 2.24(\mathrm{~s}, 3 \mathrm{H}) ;{ }^{13} \mathrm{C}-\mathrm{NMR}\left(151 \mathrm{MHz}, \mathrm{CDCl}_{3}\right) \delta 207.97$, $195.81,195.79,167.56,167.48,165.85,165.76,163.40,163.32,161.69,161.61,133.31,133.28,133.24,133.21$, $113.13,113.11,112.98,112.96,105.25,105.08,104.90,79.43,78.08,78.03,27.27$. HRMS(ESI): calcd. for $\mathrm{C}_{11} \mathrm{H}_{10} \mathrm{~F}_{2} \mathrm{NaO}_{4}\left(\mathrm{M}^{+}+\mathrm{Na}\right): 267.0439$, found 267.0446 .

(2S,3R)-1-(3,4-difluorophenyl)-2,3-dihydroxypentane-1,4-dione. Product $4 \mathrm{~m}$ was obtained as a white solid in $82 \%$ yield after flash chromatography and the enantiometric excess was determined to be $93 \%$ by HPLC analysis on Daicel Chirapak OJ-H column (hexane/isopropanol $=80 / 20$, flow rate $1.0 \mathrm{~mL} / \mathrm{min}$, $\mathrm{T}=30^{\circ} \mathrm{C}, t_{R}$ (major) $8.205 \mathrm{~min}, t_{R}$ (minor) $8.988 \mathrm{~min}$ ), m.p. $68.6-70.4{ }^{\circ} \mathrm{C} ;[\alpha]_{D}^{25}=-13.79(\mathrm{c}=0.087$, $\mathrm{CH}_{2} \mathrm{Cl}_{2}$ ) The diastereomeric ratio was determined to be 86: 14 by ${ }^{1} \mathrm{H}-\mathrm{NMR}$ on Bruker Avance 600 spectrometer; ${ }^{1} \mathrm{H}-\mathrm{NMR}\left(600 \mathrm{MHz}, \mathrm{CDCl}_{3}\right) \delta 7.86(\mathrm{t}, J=8.3 \mathrm{~Hz}, 1 \mathrm{H}), 7.82-7.75(\mathrm{~m}, 1 \mathrm{H}), 7.30(\mathrm{dd}, J=17.1$, $8.6 \mathrm{~Hz}, 1 \mathrm{H}), 5.11(\mathrm{~s}, 1 \mathrm{H}), 4.37(\mathrm{~s}, 1 \mathrm{H}), 3.91(\mathrm{~s}, 1 \mathrm{H}), 3.83(\mathrm{~s}, 1 \mathrm{H}), 2.25(\mathrm{~s}, 3 \mathrm{H}) ;{ }^{13} \mathrm{C}-\mathrm{NMR}\left(151 \mathrm{MHz}, \mathrm{CDCl}_{3}\right) \delta$ 207.73, 196.96, 155.33, 155.25, 153.61, 153.53, 151.54, 151.45, 149.87, 149.78, 131.70, 126.50, 126.47, 126.45, $126.42,118.72,118.60,118.05,117.93,79.15,75.31,27.50$. HRMS(ESI): calcd. for $\mathrm{C}_{11} \mathrm{H}_{10} \mathrm{~F}_{2} \mathrm{NaO}_{4}\left(\mathrm{M}^{+}+\right.$ $\mathrm{Na})$ : 267.0439, found: 267.0441 . 
(2S,3R)-1-(3,4-dimethoxyphenyl)-2,3-dihydroxypentane-1,4-dione. Product $\mathbf{4 n}$ was obtained as a white solid in $88 \%$ yield after flash chromatography and the enantiometric excess was determined to be 97\% by HPLC analysis on Daicel Chirapak AD-H column (hexane/isopropanol $=80 / 20 \mathrm{~min}$, flow rate $1.0 \mathrm{~mL} / \mathrm{min}, \mathrm{T}=30{ }^{\circ} \mathrm{C}, t_{R}$ (major) $14.002, t_{R}$ (minor) $15.915 \mathrm{~min}$ ), m.p. $73.0-74.6{ }^{\circ} \mathrm{C} ;[\alpha]_{D}^{25}=-14.41$ ( $\mathrm{c}=0.215, \mathrm{CH}_{2} \mathrm{Cl}_{2}$ ). The diastereomeric ratio was determined to be $92: 8$ by ${ }^{1} \mathrm{H}-\mathrm{NMR}$ on Bruker Avance 600 spectrometer; ${ }^{1} \mathrm{H}-\mathrm{NMR}\left(600 \mathrm{MHz}, \mathrm{CDCl}_{3}\right) \delta 7.64(\mathrm{~d}, J=8.4 \mathrm{~Hz}, 1 \mathrm{H}), 7.55(\mathrm{~s}, 1 \mathrm{H}), 6.94(\mathrm{~d}, J=8.4 \mathrm{~Hz}$, $1 \mathrm{H}), 5.23(\mathrm{dd}, J=5.6,4.3 \mathrm{~Hz}, 1 \mathrm{H}), 4.50-4.44(\mathrm{~m}, 1 \mathrm{H}), 4.03(\mathrm{dd}, J=10.9,6.0 \mathrm{~Hz}, 1 \mathrm{H}), 3.97(\mathrm{~s}, 3 \mathrm{H}), 3.95(\mathrm{~s}$, 3H), 3.94-3.89 (m, 1H), $2.15(\mathrm{~s}, 3 \mathrm{H}) ;{ }^{13} \mathrm{C}-\mathrm{NMR}\left(151 \mathrm{MHz}, \mathrm{CDCl}_{3}\right) \delta 207.43,197.19,154.69,149.60,127.17$, 124.17, 111.06, 110.48, 79.95, 75.40, 56.30, 56.21, 27.55. HRMS(ESI): calcd for $\mathrm{C}_{13} \mathrm{H}_{16} \mathrm{NaO}_{6}\left(\mathrm{M}^{+}+\mathrm{Na}\right)$ : 291.0839, found 291.0844 .

(2S,3R)-1-(benzo[d][1,3]dioxol-5-yl)-2,3-dihydroxypentane-1,4-dione. Product 4o was obtained as a white solid in $84 \%$ yield after flash chromatography and the enantiometric excess was determined to be $96 \%$ by HPLC analysis on Daicel Chirapak AD-H column (hexane/isopropanol $=80 / 20$, flow rate $1.0 \mathrm{~mL} / \mathrm{min}, \mathrm{T}=30{ }^{\circ} \mathrm{C}, t_{R}$ (major) $21.336 \mathrm{~min}, t_{R}$ (minor) $16.964 \mathrm{~min}$ ), m.p. $73.9-75.2^{\circ} \mathrm{C} ;[\alpha]_{D}^{25}=-9.55$ ( $\mathrm{c}=0.314, \mathrm{CH}_{2} \mathrm{Cl}_{2}$ ). The diastereomeric ratio was determined to be $93: 7$ by ${ }^{1} \mathrm{H}-\mathrm{NMR}$ on Bruker Avance 600 spectrometer; ${ }^{1} \mathrm{H}-\mathrm{NMR}\left(600 \mathrm{MHz}, \mathrm{CDCl}_{3}\right) \delta 7.61(\mathrm{~d}, J=8.2 \mathrm{~Hz}, 1 \mathrm{H}), 7.47(\mathrm{~s}, 1 \mathrm{H}), 6.90(\mathrm{~d}, J=8.2$ $\mathrm{Hz}, 1 \mathrm{H}), 6.08(\mathrm{~s}, 2 \mathrm{H}), 5.16(\mathrm{dd}, J=6.0,4.3 \mathrm{~Hz}, 1 \mathrm{H}), 4.44(\mathrm{dd}, J=6.7,4.2 \mathrm{~Hz}, 1 \mathrm{H}), 4.00(\mathrm{~d}, J=6.2 \mathrm{~Hz}$, $1 \mathrm{H}), 3.92(\mathrm{~d}, J=6.6 \mathrm{~Hz}, 1 \mathrm{H}), 2.15(\mathrm{~s}, 3 \mathrm{H}) ;{ }^{13} \mathrm{C}-\mathrm{NMR}\left(151 \mathrm{MHz}, \mathrm{CDCl}_{3}\right) \delta$ 207.41, 196.86, 153.12, 148.65, $128.85,125.88,108.66,108.42,102.33,79.77,75.46,27.55$. HRMS(ESI): calcd for $\mathrm{C}_{12} \mathrm{H}_{12} \mathrm{NaO}_{6}\left(\mathrm{M}^{+}+\mathrm{Na}\right)$ : 275.0526 , found 275.0528 .

(2S,3R)-2,3-dihydroxy-1-(6-methoxynaphthalen-2-yl)pentane-1,4-dione. Product $4 \mathbf{p}$ was obtained as a white solid in $89 \%$ yield after flash chromatography and the enantiometric excess was determined to be $97 \%$ by HPLC analysis on Daicel Chirapak IA-H column (hexane/isopropanol $=80 / 20$, flow rate $1.0 \mathrm{~mL} / \mathrm{min}$, $\mathrm{T}=30{ }^{\circ} \mathrm{C}, t_{R}$ (major) $15.424 \mathrm{~min}, t_{R}$ (minor) $18.251 \mathrm{~min}$ ), m.p. $131.2-132.3^{\circ} \mathrm{C} ;[\alpha]_{D}^{25}=-48.63(\mathrm{c}=0.183$, $\mathrm{CH}_{2} \mathrm{Cl}_{2}$ ). The diastereomeric ratio was determined to be 91: 9 by ${ }^{1} \mathrm{H}-\mathrm{NMR}$ on Bruker Avance 600 spectrometer; ${ }^{1} \mathrm{H}-\mathrm{NMR}\left(600 \mathrm{MHz}, \mathrm{CDCl}_{3}\right) \delta 8.45(\mathrm{~s}, 1 \mathrm{H}), 7.99(\mathrm{~d}, J=8.6 \mathrm{~Hz}, 1 \mathrm{H}), 7.87(\mathrm{~d}, J=9.0 \mathrm{~Hz}, 1 \mathrm{H})$, $7.81(\mathrm{~d}, J=8.6 \mathrm{~Hz}, 1 \mathrm{H}), 7.23(\mathrm{dd}, J=8.9,2.2 \mathrm{~Hz}, 1 \mathrm{H}), 7.16(\mathrm{~s}, 1 \mathrm{H}), 5.48-5.33(\mathrm{~m}, 1 \mathrm{H}), 4.55(\mathrm{dd}, J=6.4,4.0$ $\mathrm{Hz}, 1 \mathrm{H}), 4.06(\mathrm{~d}, J=6.2 \mathrm{~Hz}, 1 \mathrm{H}), 4.01-3.86(\mathrm{~m}, 4 \mathrm{H}), 2.13(\mathrm{~s}, 3 \mathrm{H}) ;{ }^{13} \mathrm{C}-\mathrm{NMR}\left(151 \mathrm{MHz}, \mathrm{CDCl}_{3}\right) \delta 207.23$, 198.41, 160.63, 138.17, 131.56, 131.11, 129.45, 127.85, 127.77, 124.87, 120.35, 106.08, 79.93, 75.80, 55.63, 27.56. HRMS(ESI): calcd for $\mathrm{C}_{16} \mathrm{H}_{16} \mathrm{NaO}_{5}\left(\mathrm{M}^{+}+\mathrm{Na}\right)$ : 311.0890, found 311.0877.

\section{Conclusions}

In summary, we have developed a highly stereoselective anti-aldol reaction of arylglyoxal monohydrates with hydroxyacetone catalyzed by quinine-derived primary amine $3 g$. The desired 2,3-dihydroxy-1,4-dione products were obtained in high yields (up to 92\%), with excellent enantioselectivities (up to $97 \%$ ee) and diastereoselectivities (up to $93: 7 \mathrm{dr}$ ).

Supplementary Materials: Supporting information including the spectrums of ${ }^{1} \mathrm{H},{ }^{13} \mathrm{C}-\mathrm{NMR}$ and HPLC are available online.

Author Contributions: Q.-X.G. and W.W. directed this project Y.-H.Z. and Y.-Z.Z. carried out all of the experiments; Z.-L.W. and T.C. finished all of the HRMS analysis. All authors have read and agreed to the published version of the manuscript.

Funding: National Natural Science Foundation of China (NSFC, 21871223), the Fundamental Research Funds for the Central Universities (XDJK2019AA003) and the Chongqing Science Technology Commission (cstccxljrc201701, cstc2018jcyjAX0548).

Conflicts of Interest: The authors declare no conflict of interest.

\section{References}

1. Mukaiyama, T. Directed aldol reaction. Org. React. 1982, 28, 203-331.

2. Mahrwald, R. (Ed.) Modern Aldol Reactions; Wiley-VCH: Weinheim, Germany, 2004; Volume 1-2. 
3. Palomo, C.; Oiarbide, M.; García, J.M. Current progress in the asymmetric aldol addition reaction. Chem. Soc. Rev. 2004, 33, 65-75. [CrossRef] [PubMed]

4. Shen, K.; Liu, X.; Zheng, K.; Li, W.; Hu, X.; Lin, L.; Feng, X. Catalytic asymmetric synthesis of 3-( $\alpha$-Hydroxy- $\beta$-carbonyl) oxindoles by a Sc ${ }^{\mathrm{III}}$-catalyzed direct aldol-type reaction. Chem. Eur. J. 2010, 16, 3736-3742. [CrossRef] [PubMed]

5. Zhao, J.; Zheng, K.; Yang, Y.; Shi, J.; Lin, L.; Liu, X.; Feng, X. Asymmetric mukaiyama aldol reaction catalyzed by $\mathrm{C}_{2}$-symmetric $N, N^{\prime}$-dioxide-Ni(II) complex. Synlett 2011, 7, 903-906. [CrossRef]

6. Hayashi, Y.; Yasui, Y.; Kojima, M.; Kawamura, T.; Ishikawa, H. Diarylprolinol in an asymmetric aldol reaction of an $\alpha$-alkyl- $\alpha$-oxo aldehyde as an electrophile. Chem. Commun. 2012, 48, 4570. [CrossRef]

7. Alberg, D.G.; Poulsen, T.B.; Bertelsen, S.; Christensen, K.L.; Birkler, R.D.; Johannsen, M.; Jørgensen, K.A. Organocatalysis with endogenous compounds: Towards novel non-enzymatic reactions. Bioorg. Med. Chem. Lett. 2009, 19, 3888. [CrossRef]

8. Hayashi, Y.; Kojima, M. Asymmetric aldol reaction of glyoxal catalyzed by diarylprolinol. ChemCatChem 2013, 5, 2883. [CrossRef]

9. Moles, F.J.N.; Guillena, G.; Nájera, C. Aqueous enantioselective aldol reaction of methyl- and phenylglyoxal organocatalyzed by N-Tosyl-( $\left.S_{a}\right)$-binam-L-prolinamide. Synlett 2015, 26, 656-660. [CrossRef]

10. Konda, S.; Guo, Q.-S.; Abe, M.; Huang, H.; Arman, H.; Zhao, J.C.-G. Organocatalyzed Asymmetric Aldol Reactions of Ketones and $\beta, \gamma$-Unsaturated $\alpha$-Ketoesters and Phenylglyoxal Hydrates. J. Org. Chem. 2015, 80, 806-815. [CrossRef]

11. Zhang, Z.-F.; Yang, X.-C.; Lu, H.-J.; Wang, M.-C. Enantioselective direct synthesis of syn- and anti- $\alpha, \beta$-dihydroxy $\gamma$-keto esters using a dinuclear zinc-AzePhenol complex. Eur. J. Org. Chem. 2018, 785-793. [CrossRef]

12. Ren, L.; Lian, X.-L.; Gong, L.-Z. Brønsted acid/Rhodium(II) cooperative catalytic asymmetric three-component aldol-type reaction for the synthesis of 3-amino oxindoles. Chem. Eur. J. 2013, 19, 3315-3318. [CrossRef] [PubMed]

13. Kano, T.; Yamaguchi, Y.; Tanaka, Y.; Maruoka, K. syn-Selective and enantioselective direct cross-aldol reactions between aldehydes catalyzed by an axially chiral amino sulfonamide. Angew. Chem. Int. Ed. 2007, 46, 1738-1740. [CrossRef] [PubMed]

14. Kano, T.; Yamaguchi, Y.; Maruoka, K. A designer axially chiral amino sulfonamide as an efficient organocatalyst for direct asymmetric anti-selective mannich reactions and syn-selective cross-aldol reactions. Chem. Eur. J. 2009, 15, 6678-6687. [CrossRef] [PubMed]

15. Xiong, Y.; Wang, F.; Dong, S.; Liu, X.; Feng, X. Asymmetric bisprolinamide-catalyzed cross-aldol reaction of aldehydes. Synlett. 2008, 1, 73.

16. Guo, Q.; Bhanushali, M.; Zhao, C.-G. Quinidine thiourea-catalyzed aldol reaction of unactivated ketones: Highly enantioselective synthesis of 3-alkyl-3-hydroxyindolin-2-ones. Angew. Chem. Int. Ed. 2010, 49, 9460-9464. [CrossRef]

17. Guang, J.; Guo, Q.; Zhao, J.C.-G. Acetylphosphonate as a surrogate of acetate or acetamide in organocatalyzed enantioselective aldol reactions. Org. Lett. 2012, 14, 3174-3177. [CrossRef]

18. Gioia, C.; Ricci, A.; Bernardi, L.; Bourahla, K.; Tanchoux, N.; Robitzer, M.; Quignard, F. Chitosan aerogel beads as a heterogeneous organocatalyst for the asymmetric aldol reaction in the presence of water: An assessment of the effect of additives. Eur. J. Org. Chem. 2013, 588-594. [CrossRef]

19. Quintard, A.; Rodriguez, J. Didecarboxylative Iron-catalyzed bidirectional aldolization towards diversity-oriented ketodiol synthesis. Chem. Eur. J. 2015, 21, 14717-14722. [CrossRef]

20. Gijsen, H.J.M.; Qiao, L.; Fitz, W.; Wong, C.-H. Recent advances in the chemoenzymatic synthesis of carbohydrates and carbohydrate mimetics. Chem. Rev. 1996, 96, 443-473. [CrossRef]

21. Enders, D.; Voith, M.; Lenzen, A. The dihydroxyacetone unit-A versatile $C_{3}$ building block in organic synthesis. Angew. Chem. Int. Ed. 2005, 44, 1304-1325. [CrossRef]

22. List, B.; Lerner, R.A.; Barbas, C.F., III. Proline-catalyzed direct asymmetric aldol reactions. J. Am. Chem. Soc. 2000, 122, 2395-2396. [CrossRef]

23. Ramasastry, S.S.V.; Zhang, H.; Tanaka, F.; Barbas, C.F., III. Direct catalytic asymmetric synthesis of anti-1,2-Amino alcohols and syn-1,2-diols through organocatalytic anti-mannich and syn-aldol reactions. J. Am. Chem. Soc. 2007, 129, 288-289. [CrossRef] [PubMed] 
24. Luo, S.; Xu, H.; Li, J.; Zhang, L.; Cheng, J.-P. A simple primary-tertiary diamine-Brønsted acid catalyst for asymmetric direct aldol reactions of linear aliphatic ketones. J. Am. Chem. Soc. 2007, 129, 3074-3075. [CrossRef]

25. Crotti, S.; Iorio, N.D.; Artusi, C.; Mazzanti, A.; Righi, P.; Bencivenni, G. Direct access to alkylideneoxindoles via axially enantioselective knoevenagel condensation. Org. Lett. 2019, 21, 3013-3017. [CrossRef]

26. CCDC 1974588 (4j) Contains the Supplementary Crystallographic Data for This Paper. Available online: www.ccdc.cam.ac.uk/data_request/cif (accessed on 29 December 2019).

27. Czarnecki, P.; Plutecka, A.; Gawroński, J.; Kacprzak, K. Simple and practical direct asymmetric aldol reaction of hydroxyacetone catalyzed by 9-amino Cinchona alkaloid tartrates. Green Chem. 2011, 13, 1280-1287. [CrossRef]

Sample Availability: Samples of the compounds are available from the authors.

(C) 2020 by the authors. Licensee MDPI, Basel, Switzerland. This article is an open access article distributed under the terms and conditions of the Creative Commons Attribution (CC BY) license (http://creativecommons.org/licenses/by/4.0/). 